\title{
La Preparación Inicial en Didáctica para la Enseñanza del Inglés en la Escuela Primaria
}

Jesús Alirio Bastidas Arteaga

Universidad de Nariño, Pasto, Nariño, Colombia

\begin{abstract}
Este estudio se realizó con el objetivo de analizar la forma en que se prepara a los estudiantes de grado de una universidad española en el componente de didáctica para la enseñanza del inglés en la primaria. Como métodos se utilizaron la investigación etnográfica y la teoría fundamentada y los datos se recogieron a través de observaciones de clase, entrevistas y análisis de documentos de un programa de grado para la formación de profesores de primaria con énfasis en lenguas extranjeras. Los resultados indicaron que la formación incluye conocimientos teóricos y prácticos mediante procedimientos sistemáticos de carácter inductivo y deductivo orientados a la comprensión y práctica de los conocimientos teóricos y varias experiencias prácticas, entre las que se destaca el micro-enseñanza apoyada en procesos de reflexión y pensamiento crítico.
\end{abstract}

\section{Introducción}

Desde finales de la década de 1980 se comenzó ha incluir el aprendizaje de las lenguas extranjeras (LE) en el nivel de primaria en muchos países del mundo, tales como en los de Europa y de Latinoamérica, fundamentados en diversas razones de tipo cultural, económico, político y educativo. Este tipo de política, además de su justificación e implementación, sin embargo, amerita la realización de investigaciones que permitan detectar el impacto que ha producido en los sistemas educativos de cada país. Por ejemplo, sería importante analizar la preparación inicial que están recibiendo los profesores de inglés para el mencionado nivel educativo en las Instituciones de Educación Superior, especialmente en el componente de Didáctica del Inglés.

La revisión de la literatura muestra que autores como Williams (1991) expresó la necesidad urgente de diseñar un modelo específico para la enseñanza del inglés a los niños, basado en la educación y en la lingüística aplicada. Bastidas (1992) propuso una preparación de los profesores de inglés para primaria basada en un enfoque teórico proveniente de la lingüística aplicada, la adquisición del lenguaje, la psicología humanística y la educación primaria; en un diseño curricular y en unos procedimientos 
metodológicos, especialmente diseñados para la enseñanza de inglés a los niños de primaria. Felberbauer (1997), también, reportó la existencia de dos modelos de enseñanza: el primero que fue diseñado por un grupo de profesores y de formadores de profesores de nueve países europeos, quienes de reunieron en Praga, después de realizar el Taller 8A en 1992. Dicho modelo incluye cuatro componentes relacionados con: educación, metodología de las lenguas extranjeras, estudios lingüísticos y culturales y psicología. El segundo modelo fue el resultado del Taller 8B que se realizó en 1995. Según Felberbauer, este modelo reflexivo afirma que el trabajo práctico de clase de los estudiantes se fundamenta con la teoría y que los conocimientos teóricos son modificados por la experiencia escolar, tal como lo muestran las flechas en la figura 1. Además, en la práctica docente, los conocimientos teóricos de los estudiantes se activan y éstos a su vez pueden afectar su desempeño en dicha práctica, tal como lo indican las flechas entrecortadas de la gráfica. Como se puede observar, el modelo incluye los mismos componentes del modelo anterior, pero además, agrega la práctica docente como la actividad central en la preparación de los profesores en ejercicio.

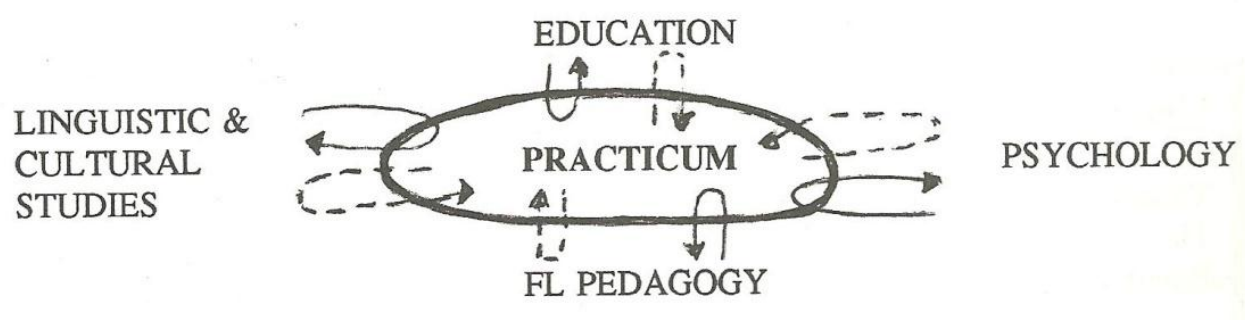

Fig. 1. Modelo de entrenamiento de profesores de lenguas extranjeras (Felberbauer, 1997).

Por otra parte, un reporte de Eurydice (2005) indica que en la mayoría de los países europeos, la preparación inicial de los profesores de LE se centra en la metodología y en la formación práctica, las cuales se desarrollan a través de cuatro actividades principales: aprendizaje de una o más LE, cursos teóricos de metodología de las LE, práctica docente y viajes cortos al extranjero. Finalmente, Bastidas (2011) ha propuesto un modelo basado en fundamentos curriculares, que incluye dos ciclos: uno de desarrollo de la lengua extranjera hasta alcanzar un nivel, al menos, de intermedioalto o B2 según el Marco Común Europeo, y un ciclo de preparación profesional en educación en LE para primaria, el cual en su fundamentación teórica incluye 
conocimientos sobre aprendizaje del lenguaje (AL), AL según el contexto, AL a través de la enseñanza y desarrollo professional inicial, y en el componente práctico e investigativo propone la realización de actividades observacionales, prácticas, experienciales e investigaciones de campo en la escuela primaria desde el primer semestre del segundo ciclo, de tal manera que los estudiantes comiencen a comprender, interpretar e idealmente a construir teoría a partir de la práctica. Este modelo, lo podemos visualizar en el siguiente diagrama:

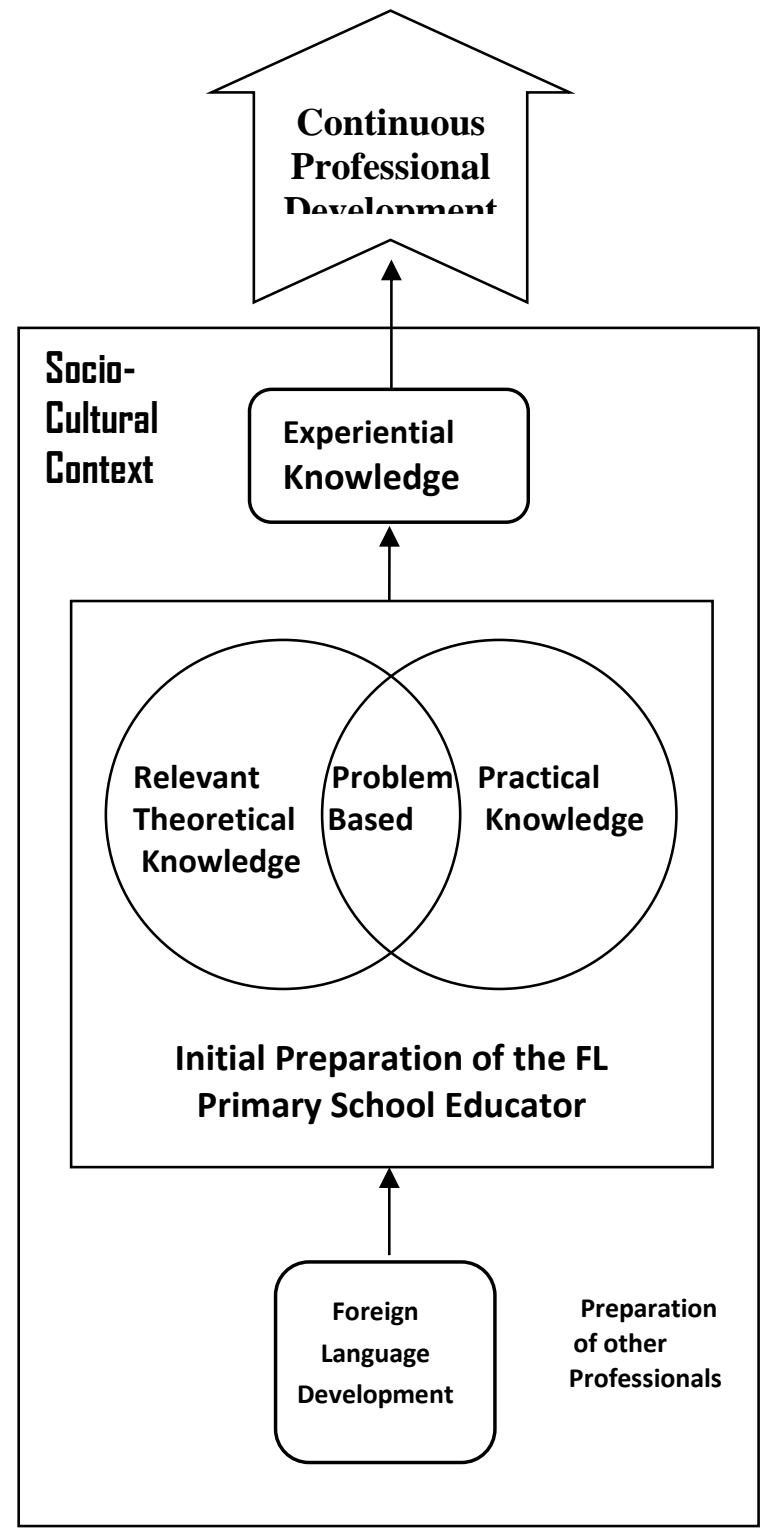

Fig. 2. Modelo basado en el currículo para la educación de profesores de lenguas extranjeras de primaria. (Bastidas, 2011) 
Puesto que el interés de esta investigación se centró en la formación didáctica de profesores de inglés para la educación primaria hay necesidad de delimitar el alcance del componente didáctico. Al respecto, Williams (1991) llamó la atención sobre la necesidad de "diseñar un modelo didáctico específico para la enseñanza del idioma inglés a los niños más pequeños, a diferencia de aquéllos otros que se limitan a transferir los esquemas de enseñanza a jóvenes o adultos para aplicarlos a las primeras etapas”(citado en Durán, Sánchez-Reyes y Beltrán, 2004: p. 53), lo cual corrobora lo expresado por autores clásicos como Mackey, quien se refirió a la importancia de la edad con relación al aprendizaje y al método:

One cannot expect a child of fifteen to learn in exactly the same way as a child of five. A learner's age affect the stability of a method in (1) content and (2) presentation and practice. (1965: p. 325)

Lo anterior significa que el componente metodológico de un programa de formación de profesores de inglés para primaria deberá tener en cuenta al niño, según su edad en el nivel de primaria como el sujeto, en sus aspectos cognitivos, lingüísticos, afectivos, físicos y socio-culturales, para quien se orienta la metodología, sin desconocer que se deban tener en cuenta algunos aspectos generales, no solamente de la Metodología de la Enseñanza de las Lenguas Segundas o Extranjeras (MELSE), sino también de la pedagogía de la enseñanza primaria, tal como lo indica Brewster (1991).

Caracterización. El componente de MELSE se centra en el 'cómo' enseñarlas, es decir, en los procedimientos, técnicas y materiales de enseñanza. Uno de los pioneros en identificar el contenido y campo de acción de la MELSE fue Mackey (1965), quien se refirió a las variables que conforman este campo, así: método, materiales, maestro e instrucción. Por otra parte, Richards, Platt \& Platt (1992) definen a la metodología como al estudio de las prácticas y los procedimientos que se usan para enseñar, así como también a los principios y creencias que las subyacen (p. 228).

Propósito del Componente Metodológico. Varios autores se han referido al propósito y los objetivos del componente metodológico para la formación de profesores de lenguas en general, tales como Wallace (1991) y Richards (1998). Específicamente para la educación primaria, Felberbauer (1997) se refiere a los objetivos del componente metodológico, los cuales cubren desde el desarrollo de habilidades y actividades profesionales, hasta la creación de situaciones de aprendizaje de una lengua en las clases 
de primaria. Luego, indica objetivos para el desarrollo de habilidades metodológicas, de adaptación de recursos y materiales, de uso de los medio (media) y de manejo de textos apropiados a la edad de los niños.

En su propuesta de cursos de Lengua Inglesa y de Didáctica, Durán, SánchezReyes y Beltrán (2004), proponen: la realización de programas analíticos; evaluación de recursos y materiales didácticos, fuentes bibliográficas y propuestas metodológicas y de política legislativa; diseño, adaptación y explotación pedagógica de materiales, recursos y actividades de enseñanza y para el "manejo de una variedad de estrategias de dinámica de grupos y de adopción del rol adecuado del docente para una situación dada”. (p. 99).

Contenido del componente metodológico. Por la caracterización anterior y por tradición la MELSE incluiría aspectos relacionados con la práctica y en consecuencia sus temas centrales serían los métodos, los materiales, los procedimientos y técnicas y el papel del profesor, los cuales se desarrollan a través de cursos, seminarios o talleres en la Universidades y la práctica docente que realizan los estudiantes en las escuelas. Con relación a la MELSE, el Modelo de Praga (Ferbelbauer, 1997) propone la inclusión de los siguientes aspectos como parte de este componente: metas y objetivos, métodos y procedimientos, media y evaluación. Otros autores como Durán, Sánchez-Reyes y Beltrán (2004), también incluyen aspectos relacionados con el diseño de syllabus o contenidos programáticos. Recientemente autores como Madrid y McLaren (2004) acogen el modelo de Stern (1983), como base para lo que ellos denominan Didáctica de las Lenguas Extranjeras, la cual en su componente práctico incluye temas como: currículo de las LE, metodología, políticas y organización del sistema educativo y entrenamiento de profesores.

En conclusión, se observa una tendencia de la muestra de autores referenciados a centrar la metodología en el 'cómo enseñar' y a cubrir aspectos relacionados con la preparación para enseñar (planeación), la enseñanza como tal (métodos, procedimientos, técnicas y materiales) y la evaluación de lo aprendido. Puesto que en la introducción de esta sección se ha expresado la necesidad de acogerse a un marco conceptual para la enseñanza de las LE a los niños, en este caso de Educación Primaria, la metodología debe incluir temas específicos para niños de dicho nivel educativo acorde con el rango de edad. 
La revisión de la literatura anterior muestra que se ha avanzado en el diseño de modelos de preparación de profesores para la enseñanza del inglés en primaria, así como también en la caracterización, objetivos, contenidos y procedimientos del componente didáctico; sin embargo, en la literatura revisada no hemos encontrado resultados de investigaciones que exploren, describan, analicen, expliquen, o evalúen los modelos en general y el componente didáctico en particular. En consecuencia, este estudio se realizó con el objetivo de dar respuesta a la pregunta: ¿Cómo se prepara a los estudiantes de grado de una universidad española en el componente de didáctica para la enseñanza del inglés en la escuela primaria?

\section{Metodo}

La investigación ${ }^{1}$ se orientó bajo los principios de la investigación cualitativa, cuya importancia radica en que se concentra en los procesos, motiva la descripción detallada de los fenómenos, se interesa por el sentido que los participantes le dan a sus acciones, obliga al investigador a realizar trabajo de campo e implica unos procedimientos de carácter inductivo.

Acorde con el anterior enfoque, se utilizó la investigación etnográfica y la Teoría Fundamentada. La primera tiene como objetivo describir e interpretar los comportamientos culturales, creencias, sentimientos, etc., de un grupo de personas y además se caracteriza por ser de carácter "emica", es decir, por buscar una mirada y una interpretación desde la perspectiva de los participantes, por ser holística, es decir, por mirar los fenómenos en contexto y teniendo en cuenta el todo de una realidad, por tener en cuenta los factores de tipo socio-cultural que rodean al fenómeno objeto de estudio y por orientarse a grupos de personas. La Teoría Fundamentada es un método de recolección y análisis de información para describir, interpretar y sobre todo explicar un fenómeno de la realidad social. Su propósito es formular teorías con base en los datos recogidos en el campo, pero para ello, comienza por el nivel de la descripción a través de la generación de conceptos, que son los aspectos iniciales que se utilizaron en esta investigación (Andréu, García-Nieto y Pérez, 2007).

Las técnicas de recolección de información utilizadas fueron las observaciones de 41 horas de clase, cuatro entrevistas a las profesoras y el análisis documental, las cuales están acorde con el paradigma y los tipos de investigación seleccionados. 
Los participantes en esta investigación fueron los estudiantes de los dos cursos de Didáctica de la Lengua Inglesa de pregrado y las dos profesoras de dichos cursos de un programa de grado de una universidad española.

\section{Resultados y Discusión}

El análisis de los datos se realizó a través de las siguientes etapas: organización de los datos, conceptualización, categorización, interpretación y reporte escrito de los resultados (Bastidas, 2002). Así mismo, se utilizaron diferentes tipos de codificación propuestos por autores como Strauss y Corbin (1990), Charmaz (2006) y Saldaña (2009), los que fueron muy útiles para la obtención de los siguientes resultados, que se reportan según el pregunta central y las categorías y subcategorías descubiertas a través de las etapas de la Teoría Fundamentada.

¿Cómo se prepara a los estudiantes de grado de una universidad española en el componente de didáctica para la enseñanza del inglés en la escuela primaria? es la pregunta central de este estudio y cuyos datos resultado de las observaciones de clase y de las entrevistas a las profesoras nos permitieron generar las siguientes categorías, subcategorías y conceptos.

\section{Proceso de formación didáctica para la enseñanza del inglés en la escuela primaria}

Puesto que esta categoría responde a la pregunta ¿cómo?, la podemos definir como al conjunto de métodos, procedimientos y técnicas para la enseñanza de los conocimientos teóricos y prácticos de la didáctica de la lengua inglesa.

Teniendo en cuenta que en la preparación didáctica de los estudiantes se incluye conocimientos teóricos y prácticos (subcategorías), los cuales fueron identificados a través de las observaciones, de las entrevistas y de los programas de los cursos de didáctica, a continuación me refiero a los procedimientos específicos utilizados por los profesores para la enseñanza de cada uno.

Procedimientos para la enseñanza de conocimientos teóricos: Los datos recogidos de las 15 observaciones del curso de DLI 1 muestran el predominio de un 
método inductivo en la presentación y práctica de los conocimientos teóricos del curso. Es decir, que la profesora inició con el uso de cuestionarios, actividades (tasks) y guías sobre cada uno de los temas teóricos, para con base en ellas hacer una serie de preguntas que auscultaban los conocimientos previos de los alumnos, así como también sus conocimientos provenientes de las lecturas previas para luego hacer resúmenes o síntesis de los conceptos teóricos. Veamos la descripción de una de las clases realizadas por la profesora del curso:

La profesora parte de las creencias comunes que tienen los estudiantes y desde luego la mayoría de personas, las cuales demuestran variedad de opiniones y en algunos casos mayor consenso entre los alumnos. Con base en dichas creencias reflejadas en las respuestas de los alumnos a un cuestionario elaborado por Lightbown y Spada (1999), la profesora (P) estimula a los estudiantes (Es) para que sustenten sus respuestas y no se limiten a decir cual respuesta seleccionaron. A través de preguntas, la P también genera dudas en los Es, quienes las demuestran quedándose callados y contestando, pero no en forma clara; momento en el cual la P les solicita que sean precisos o que expliquen mejor sus respuestas.

Las preguntas de la $P$ también permiten descubrir en los Es, especialmente, en un Es de Checoslovaquia y dos de España, algunos conocimientos previos que es posible los hayan adquirido en otras materias, estudiadas previamente. Ante las respuestas de los Es, la P procede a clarificar dichos conceptos para toda la clase o a sustentarlos, acorde con su conocimiento e intención.

Otro mecanismo que usa la $P$ es ayudar a los Es a entender aquéllos conceptos que pueden ser nuevos para la mayoría, ya sea por medio de definiciones, de ejemplos o de experiencias personales o de los Es. Luego la P continúa con el proceso de preguntas, lo cual pone a pensar a los Es y a reflejar su pensamiento o razonamiento a través de sus respuestas. Así mismo, ante algunas afirmaciones de los autores y de las preguntas de la $P$, quien los guía para que utilicen su experiencia de aprendizaje del lenguaje como medio para responder, una estudiante argumenta su concepto basado en su experiencia, la cual le ha permitido afirmar que a pesar de haber estudiado inglés desde prescolar, al final de la primaria "no aprendió nada". (Esta afirmación, pone en duda el concepto de varios expertos en SLA que han afirmado que "the earlier the better"). Ante lo cual, la P explica que efectivamente, el problema no está en que se comience muy temprano, ni con extensión en el tiempo, sino que el aprendizaje de las lenguas depende de la calidad del contenido y de la intensidad en el aprendizaje. Finalmente, la $P$ guía a los Es a pensar en los conceptos de Adquisición del Lenguaje para su posible aplicación en el salón de clase, ante lo cual los Es reflejan sus dudas, quizá porque aún les falta un mayor conocimiento metodológico de la enseñanza de las lenguas y desde luego de experiencia docente. (En resumen, el conocimiento practicado en la mayor parte de tiempo de esta clase ha sido de carácter teórico, sobre conceptos provenientes del campo de Adquisición de las Lenguas). 
Por otra parte, los datos recogidos de 18 (de las 26) observaciones realizadas en el curso de DLI 2, dedicadas a temas de carácter teórico, demostraron el predominio de un método deductivo en la presentación y práctica de los conocimientos teóricos del curso. Esto significa, que la profesora o los estudiantes iniciaron con la explicación de los temas teóricos, para luego proceder a realizar actividades de carácter grupal con el objeto de que los estudiantes entiendan y asimilen dichos conceptos. El análisis de los datos precisamente permitió sustentar las siguientes sub-subcategorías:

1. Explicación de un tema teórico, la que incluyó los siguientes conceptos: especificación del procedimiento seguido por la profesora, indicación de la importancia del tema, referencia a la aplicabilidad del tema en las escuelas e invitación a los estudiantes a ser críticos, y

2. Acciones para guiar a los estudiantes al entendimiento y a la asimilación de los conocimientos teóricos, entre las que se observaron: estructuración de trabajo en grupo, indicación del objetivo de la actividad, identificación del tipo de actividad, realización de la actividad, reporte de resultados de la actividad por parte de los estudiantes, sugerencias pedagógicas por parte de la profesora y demostración de una actitud crítica por parte de los estudiantes.

Es importante, además, destacar que la profesora delegó en sus estudiantes la responsabilidad de hacer presentaciones sobre algunos temas teóricos, cuya descripción de una de las presentaciones mostró lo siguiente:

El análisis de las sub-categorías nos muestra la sustentación de una en particular, que en esta clase la he denominado: ayudando a los estudiantes a entender conocimiento teórico. Esta denominación la utilizo por cuanto ahora son los estudiantes, quienes han presentado los temas, lo cual significa que los expositores, han tenido que buscar la información, ordenarla en un power point, seleccionar materiales y acordar como hacer la presentación. Es decir, que la profesora ha delegado su responsabilidad en los estudiantes para que, posiblemente, aprendan más de los temas y practiquen como hacer exposiciones, es decir, jugar el papel de profesores o expositores y al mismo tiempo revisar su inglés y ponerlo a prueba en clase. Esto, desde luego, genera mucho estrés en algunos estudiantes, quienes lo reflejan en sus exposiciones, (pero que afortunadamente, se aminora un poco por el apoyo de los compañeros, dada la responsabilidad grupal de los mismos). Sin embargo, esta sub-categoría se relaciona con la subcategoría generada en tres clases anteriores y que la denominé: proporcionando conocimiento teórico, denominación que decidí mantener y que, a su vez, se ha sustentado en dos sub-subcategorías: explicando tópicos teóricos y analizando las 
presentaciones, las cuales se han sustentado en 11 y 10 conceptos respectivamente. Dichos conceptos han girado alrededor de la forma como se realizan las explicaciones y del tipo de conocimiento teórico que se ha presentado en la primera sub-subcategoría.

Mientras que en la segunda, hemos encontrado conceptos que han girado alrededor de manejo de clase por parte de la profesora, promoviendo una actitud crítica en los estudiantes y haciendo sugerencias a los estudiantes sobre aspectos metodológicos.

Procedimientos para la enseñanza de conocimientos prácticos: $\mathrm{El}$ análisis de los datos provenientes de las observaciones nos indica que en los dos cursos se utilizaron diferentes actividades para el desarrollo del conocimiento práctico de los estudiantes. En el curso de Didáctica de la Lengua Inglesa I se destacaron dos tipos de actividades de carácter práctico: lectura oral de cuentos infantiles y demostración de métodos de enseñanza.

La subcategoría denominada práctica de una estrategia de enseñanza para niños se sustentó con un mayor número de conceptos, debido que hubo dos demostraciones en algunas de las clases y también hubo tiempo para feedback de la profesora. Esta categoría se enriqueció con varios conceptos, lo que indica que los alumnos necesitan de mayor retro-alimentación para hacer una demostración como la profesora lo había planeado. En una de las clases, por ejemplo, aparecieron conceptos de sugerencias para mejorar la actividad y la respectiva felicitación a los estudiantes por la demostración realizada, dada la naturaleza de las historias escogidas y el esfuerzo realizado por ellos. Lo que quedó en duda fue la adaptación del lenguaje para asegurar la comprensión de los niños de primaria.

La sub-categoría demostrando métodos de enseñanza de lenguas extranjeras me permitió identificar varios conceptos, aunque con mayor riqueza en la primera demostración que en las dos restantes. Es de destacar la insistencia de la profesora en demostrar que con un método como el TPR es factible enseñar una L2 o LE sin necesidad de recurrir a la L1, ya que según ella, los estudiantes comprendieron todo lo enseñando en checo. Personalmente, considero que el método es interesante y su sencillo procedimiento funciona con los niños, pero para lograr unos resultados mucho más productivos necesita ser combinado con otros métodos. Por otra parte, las acciones pueden ser entendidas por los niños que estén muy atentos, pero los demás, corren el 
riesgo de hacer las cosas mecánicamente, es decir por imitación. Esta sub-categoría refleja la preocupación de la profesora por proporcionar a los estudiantes una experiencia práctica en la utilización de un método específico para los niños.

Sin embargo, es importante señalar la identificación de problemas de pronunciación y fluidez de los estudiantes, los cuales se dan en un contexto, donde los estudiantes han tenido tiempo para prepararse, exclusivamente en la lectura del cuento, pero aun así, en los cuatro estudiantes que realizaron esta actividad se detectó problemas similares. Este aspecto puede servir de sustentación a la duda que he tenido sobre el nivel de dominio del inglés de los estudiantes como un factor que puede estar interfiriendo en la comprensión de los temas teóricos que se presentan en clase; además, no podemos asumir, que por el solo hecho de enseñar contenidos a través del inglés, automáticamente los estudiantes van a mejorar su nivel de lengua. Al respecto, tengo la hipótesis, de que los estudiantes, al menos deben haber alcanzado un nivel intermedioalto de dominio del inglés para poder procesar y aprender conocimientos teóricos o contenidos de diferentes áreas del conocimiento, incluidos aquéllos de la Didáctica de las LE.

Los datos recogidos en las observaciones del curso de DLI 2 me permitieron sustentar la sub-categoría denominada obteniendo conocimiento basado en la experiencia inicial, la cual incluyó las sub-subcategorías denominadas: demostración de micro-clases y evaluando las demostraciones de micro-enseñanza. Los conceptos de la primera sub-subcategoría mostraron una gran riqueza de información, representada en la cantidad de acciones e interacciones que suceden en este tipo de actividad. Ésta se sustentó en una cantidad de conceptos que se acumularon más en el concepto sobre práctica del nuevo tema, el cual parece ser muy rico en el campo de la metodología de la enseñanza de las lenguas, dada su naturaleza de ejercicios, actividades o tareas (tasks). Por otra parte, al parecer algunos de los estudiantes las tomaron como centro de su clase, ante lo cual surge la pregunta, ¿significa esto, que para algunos estudiantes una micro-clase (y después en su vida profesional) una clase es igual a un conjunto de técnicas y de ejercicios?

Los otros conceptos corresponden a las formas de introducir una clase, explicación del nuevo tema, formas de terminar una clase y manejo de clase. Sin embargo, aquí se observaron algunas particularidades. En primer lugar, no se observó 
repetición de actividades, es decir, en cada micro-clase aparecieron ciertas acciones diferentes, lo que permitió ampliar el número de conceptos. La introducción de clase y la forma de terminar una clase fueron las más cortas en el tiempo, pero sin embargo, aparecieron acciones diferentes en todas las micro-clases. La explicación del nuevo tema no se realizó en algunas micro-clases, quizá porque los estudiantes asumieron que ésta ya se había realizado en una clase anterior. La de manejo de clase, también se sustentó con varias acciones, lo cual puede haber sido el resultado de las acciones de los estudiantes, a quienes seguramente se los sugirió por parte de la profesora que realicen determinado "mal comportamiento", con el objeto de ver cómo reacciona el profesor. Finalmente, un momento de clase que no se mostró en la mayoría de micro-clases fue el correspondiente al repaso, el cual he observado que ha ido desapareciendo en las etapas de las clases reales de los profesores. Sin embargo, hay autoridades del campo que indican que esta es una fase muy importante, precisamente para contrarrestar el fenómeno del olvido. Es decir, que entre más temprano y más frecuente se hagan los repasos, mayor posibilidad de retener y de internalizar los conocimientos. La segunda sub- subcategoría: evaluando las demostraciones de micro-enseñanza, se resalta en el componente práctico, debido a la estructuración y al manejo organizado y estricto que le da la profesora, con lo cual ha logrado realizar un interesante proceso de evaluación, que incluye auto-evaluación personal, evaluación de la profesora y evaluación de los compañeros.

Como se puede observar las dos profesoras han utilizado diferentes procedimientos y actividades para la preparación de los estudiantes en el componente didáctico, los cuales coinciden con aquéllos que han propuesto varios autores. Para la preparación de los estudiantes en conocimientos teóricos, lo típico es el uso de la conferencia o exposición oral del profesor, quizá acompañada de tutorías esporádicas o en el otro extremo el uso de tutorías individuales acompañadas de conferencias opcionales (Wallace, 1991). Sin embargo, tal como lo expresa Wallace, existe una diversidad de procedimientos generales utilizados a nivel universitario en diferentes ramas del conocimiento que deberían incluirse en el componente didáctico de las LE, tales como: el seminario, la lectura guiada ${ }^{2}$, la técnica socrática ${ }^{3}$. Richards (1998), también, recomienda el uso de discusiones y el trabajo en grupos pequeños. Además, Woodward (1992) se refiere a diferentes tipos de conferencias, tales como: conferencia 
buzz-group ${ }^{4}$, conferencia con palabras clave ${ }^{5}$, conferencia estilo Curran $^{6}$ y retroalimentación con base en cuestionarios ${ }^{7}$.

Si bien los resultados del estudio mostraron que la formación en conocimientos teóricos incluyó diferentes procedimientos y actividades y que la revisión de la literatura demuestra la existencia de una variedad de actividades o estrategias de enseñanza, parece que el procedimiento se centrase en las dos primeras fases de una lección: la de la presentación o explicación de los conocimientos teóricos y la de la práctica a través de diferentes actividades realizadas por los alumnos con el objeto de que éstos comprendan y asimilen los conocimientos teóricos. Sin embargo, este procedimiento, quedaría incompleto, si no se continúa con una siguiente etapa, que algunos autores como de Mattos (1974) la denominan "la fase de la integración y de la fijación del contenido del aprendizaje". Esta es muy importante para la activación de dos procesos cognitivos esenciales en el aprendizaje: la organización y la integración (Mayer, 2008). El primero consiste en la combinación, conexión y organización de la información verbal y visual relevante para formar una totalidad integrada en forma coherente. Para el efecto, se pueden realizar actividades como: cuadros sinópticos, mapas mentales o conceptuales, identificación de las estructuras retóricas de un texto, preguntas-guía, etc. El proceso de integración consiste en conectar la información organizada en la memoria de trabajo al conocimiento previo, de tal manera que se constituya en un nuevo conocimiento integrado en forma coherente, el cual se depositará en la memoria a largo plazo. Si el estudiante logra hacer esta integración habrá una buena retención y le permitirá hacer una buena transferencia de los conocimientos teóricos a diferentes contextos, logrando así el aprendizaje de los mismos (Mayer, 2008). Para el logro del objetivo de este proceso se recomienda el uso de cuadros sinópticos previos, de preguntas, de resúmenes y de actividades que fomenten la reflexión y la crítica, entre otras.

Con relación a la preparación de los estudiantes en los conocimientos prácticos, además de las actividades de demostración de métodos, de lectura oral de cuentos infantiles, de uso de las TICs, de trabajos por proyectos y de la micro-enseñanza, se propone la realización de simulaciones, juegos de roles, estudios de campo y estudios de casos (Wallace, 1991). Además, se recomienda la realización de observaciones, tanto en la universidad, como en las escuelas de primaria. En la universidad se puede observar 
videos de clases, demostraciones de los profesores y micro-clases de los compañeros, y en las escuelas de primaria se pueden implementar actividades de observación del contexto escolar, de uno o más estudiantes en sus actividades de clase y de las clases en general. Además, es importante realizar estas actividades en forma progresiva, así: actividades de observación, actividades de ayudantía a los profesores de primaria y finalmente, actividades de práctica pedagógica, las cuales deben acompañarse de los procesos de reflexión y crítica, tal como lo realiza la profesora de DLI 2 en la microenseñanza y que ha sido sustentada por diferentes autores, como: Barlett (1990), Wallace (1991), Richards y Lockhart (1994) y Richards (1998).

\section{Conclusiones y recomendaciones}

La inclusión de las lenguas extranjeras, en particular del inglés, en la primaria de los sistemas educativos de varios países de Europa y de otros continentes a partir de la década de 1980 obligó a las instituciones reguladoras de la educación y a las universidades a implementar programas de desarrollo o formación profesional, tanto para los profesores de primaria, como para la preparación de nuevos profesores. En este último caso, las universidades europeas, por ejemplo, han ofrecido programas de grado para la educación primaria incluyendo un énfasis en la enseñanza de las lenguas extranjeras, tal como sucedió con la universidad española, objeto de este estudio, cuyo programa de grado se inició en 1992.

Puesto que han transcurrido más de 30 años, después de la implementación de la anterior iniciativa es necesario sistematizar los conocimientos y experiencias al respecto, ya sea para elaborar el estado del arte o para promover investigaciones, las cuales parecen ser escasas hasta el momento. En este marco, se ha realizado el presente estudio cuya pregunta central de investigación fue ¿Cómo se prepara a los estudiantes de grado de una universidad española en el componente de didáctica para la enseñanza del inglés en la educación primaria?

Los resultados indican que esta preparación incluye una serie de conocimientos teóricos y de experiencias prácticas relacionadas con la metodología de la enseñanza del inglés en general (Wallace, 1991; Richards, 1998), así como también con la metodología de la enseñanza del inglés para primaria (Bastidas, 1992; Felberbauer, 1997; Vez, 2002; Pérez y Roig, 2004a; Pérez y Roig, 2004b). Con relación a la preparación inicial en los conocimientos teóricos, ésta se realiza a través de 
procedimientos deductivos e inductivos bastante claros y organizados que incluyen una variedad de actividades para facilitar las fases de la presentación y práctica de los temas de cada clase. Sin embargo, se recomienda la inclusión de actividades adicionales con el objeto de que los estudiantes logren organizar los nuevos conocimientos e integrarlos con los conocimientos previos en su memoria a largo plazo. Según Mayer (2008), estos dos procesos cognitivos son esenciales para asegurar el aprendizaje. Además, es urgente la revisión de las políticas institucionales con relación al nivel de inglés de los estudiantes, como pre-requisito para ingreso al programa y para tomar los cursos de didáctica. Al respecto se observó que para varios de ellos, especialmente en el cursos de Didáctica 1, éste se convirtió en un factor de insatisfacción, tanto para la profesoras como para los estudiantes, dado su grado de interferencia para entender y mucho más para asimilar y hacer uso creativo de los conocimientos, tanto teóricos como prácticos.

Para la preparación en conocimientos prácticos se encontró que las profesoras han incorporado en los dos cursos de didáctica experiencias interesantes, como: la demostración de métodos, la lectura de cuentos infantiles, el trabajo por proyectos, el uso de las TICs y la micro-enseñanza, esta última acompañada de la reflexión y la crítica por parte de los estudiantes y de la profesora, con lo cual se proporciona a los estudiantes la adquisición de un conocimiento 'experiencial, tal como lo propone Wallace (1991). Finalmente, se recomienda la revisión de la relación entre las experiencias prácticas y los conocimientos teóricos, de tal manera que los estudiantes los puedan articular en forma coherente y desarrollen aún más su capacidad reflexiva y crítica.

Puesto que el área de formación inicial y de desarrollo profesional de los profesores de inglés para la educación primaria no demuestra mayor desarrollo en el aspecto investigativo, se recomienda la réplica de este tipo de estudios no solamente en el componente didáctico, sino también en todos los componentes de los programas de preparación de profesores de lenguas extranjeras para la educación primaria, los cuales seguramente promoverán estudios de evaluación del impacto y de la efectividad de este tipo de programas profesionales.

\section{Referencias}

Andréu, J., García-Nieto, A., y Pérez, A. (2007). Evolución de la Teoría fundamentada como técnica de análisis cualitativo. Madrid, España: CIS. 
Barlett, L. (1990). Teacher developmente through reflective teaching. In J. Richards, \& D. Nunan. (Eds.). Second language teacher education (pp. 202-214). Cambridge: Cambridge University Press.

Bastidas, J.A. (2002). El análisis de datos en la investigación cualitativa. Revista de Investigaciones, 11, (1). 28-41.

Bastidas, J.A. (1992). English in primary school: Pros and cons of the new Colombian law. Lecture given at the $27^{\text {th }}$ ASOCOPI Convention. Universidad del Valle, Cali, November 1. (Publicada en J. A. Bastidas. (2002). Teoría y práctica de la enseñanza del inglés, (pp. 158-183). Tunja: La Estación.

Bastidas, (2011). Educating foreign language primary school teachers: A review of models of teacher education and new proposal. Bellaterra Journal of teaching \& Learning Language \& Literature, Vol. 4(1), p. 1-16.

Brewster (1991). What is good in primary practice. In C., Brumfit, J., Moon, \& R., Tongue. (Eds.). Teaching English to children: From practice to principle. (pp. 1-17). London: Collins ELT.

Charmaz, K. (2006). Constructing grounded theory: A practical guide through qualitative analysis. Los Angeles, CA: Sage Publications Ltd.

de Mattos, L. A. (1974). Compendio de didáctica general. Buenos Aires: Editorial Kapeluz.

Doyé, P., \& Hurrell, A. (Eds.). (1997). Foreign language learning in primary school. Strasbourg Cedex, Germany: Council of Europe Publishing.

Durán, R., Sánchez-Reyes, P., y Beltrán, F. (2004). La formación del profesorado de Lengua inglesa en un contexto europeo. Salamanca, España: Ediciones ALMAR.

Eurydice. (2005). Cifras clave de la enseñanza de lenguas en los centros escolares de Europa. Madrid: Eurydice Unidad Española.

Felberbauer, M. (1997). Teacher education. In P., Doyé \& A., Hurrell. (Eds.). Foreign language learning in primary school (p. 77-85). Strasbourg Cedex, Germany: Council of Europe Publishing.

Lightbown, P. M., \& Spada, N. (1999). How languages are learned. Oxford: Oxford University Press.

Madrid, D., y McLaren, N. (Eds.). (2004). TEFL in primary education. Granada, España: Universidad de Granada.

Mackey, W. F. (1965). Language teaching analysis. London: Longman.

Mayer, R. (2008). Learning and instruction. Columbus, OH: Pearson - Merrill Prentice Hall.

Perez, P., \& Roig, V. (2004a). Enseñar y aprender inglés en educación infantil y primaria. Vol. I. Barcelona: Horsori Editorial, S.L.

Perez, P., \& Roig, V. (2004b). Enseñar y aprender inglés en educación infantil y primaria. Vol. II. Barcelona: Horsori Editorial, S.L.

Richards, J. (1998). Beyond training. Cambridge: Cambridge University Press.

Richards, J., \& Lockhart, C. (1994). Reflective teaching in second language classrooms. New York: Cambridge University Press.

Richards, J., Platt, J., \& Platt, H. (1992). Longman Dictionary of second language teaching and applied linguistics. Essex, UK: Longman Group Limited.

Saldaña, J. (2009). The coding manual for qualitative researchers. Los Angeles, CA: Sage Publications. 
Stern, H. H. (1983). Fundamental concepts of language teaching. Oxford: Oxford University Press.

Strauss, A., \& Corbin, J. (1990). Basics of qualitative research: Grounded theory procedures and techniques. Newbury Park, CA: Sage Publications.

Vez, J. M (Ed.). (2002). Didáctica de la lengua extranjera en educación infantil y primaria. Madrid: Editorial Síntesis, S.A.

Wallace, M. J. (1991). Training foreign language teachers: A reflective approach. Cambridge: Cambridge University Press.

Williams, M. (1991). A framework for teaching English to young learners. In In C. Brumfit, J., Moon, \& R., Tongue. (Eds.). Teaching English to children: From practice to principle (pp. 1-17). London: Collins ELT.

Woodward, T. (1992). Ways of training. Harlow: Longman.

\section{Referencias del autor:}

Jesús Alirio Bastidas Arteaga es profesor en la Universidad de Nariño, Pasto, Nariño, Colombia. Es Doctor en Lengua, Literatura y Aprendizaje (1999) y tiene un Máster en Lectura y Escritura (1996) otorgado por la Universidad del Sur de California, Los Angeles, CA, EE.UU. (LASPAU-Fulbright Scholar). También tiene un máster en Lingüística: TEFL (1983) de la Ohio University, Athens, OH, EE.UU. (Fulbright Scholar). Ha completado un Curso de Postgrado en ELT (1992) en Thames Valley University, Londres, Inglaterra (British Council Scholar). Fue profesor visitante en la Universidad Central de Washington, (2008) e hizo estudios post-doctorales en Didáctica de la Lengua y Literatura en la Universidad Autónoma de Barcelona (Erasmus-Mundus Scholar) durante el curso 2010-2011.

Email: jabas3@yahoo.es

\section{Para citar este artículo:}

Bastidas Arteaga, J-A. (2013). La preparación inicial en didáctica para la enseñanza del inglés en la escuela primaria. Bellaterra Journal of Teaching \& Learning Language \& Literature, 6(1), 1-17.

\footnotetext{
${ }^{1}$ Este artículo es un reporte de la investigación realizada en calidad de becario Erasmus Mundus, bajo la asesoría de la Dra. Melinda Dooly durante la realización de mis Estudios Post-Doctorales en el Programa de Doctorado en Didáctica de la Lengua y la Literatura.

${ }^{2}$ A los estudiantes se les asigna un capítulo de un libro o un artículo para que lo lea con un propósito general o específico.

${ }^{3}$ El profesor guía a los estudiantes para entender un tema o una verdad a través de una serie de preguntas.

${ }^{4}$ En grupos pequeños, los estudiantes escuchan una conferencia y en determinado tiempo presentan a la clase las ideas principales.

${ }^{5}$ Con anticipación a los estudiantes se les entrega una lista de palabras y frases importantes sobre una conferencia para que ellos hagan predicciones sobre su significado. Durante la conferencia toman nota sobre la forma en que fueron utilizadas las palabras y frases y luego las reportan en el momento de la discusión.

${ }^{6}$ Dos estudiantes se sientan detrás del profesor en determinados momentos de la conferencia reportan a la clase la ideas principales de la conferencia.

7 Inmediatamente se termina la conferencia, los estudiantes completan un cuestionario con preguntas, tales como: "Ahora he aprendido ...", "Ahora puedo enumerar ...", "No estoy aún seguro de ...", para entregarlas al profesor o para discutirlas luego.
} 